\title{
VARIATIONAL INEQUALITIES AND FREE BOUNDARY PROBLEMS ${ }^{1}$
}

\author{
BY DAVID KINDERLEHRER
}

The study of variational inequalities and free boundary problems finds application in a variety of disciplines including physics, engineering, and economics as well as potential theory and geometry. In this brief informal exposition, we intend to discuss several examples with the intention of illustrating the questions and ideas which comprise this theory. A general introduction to variational inequalities is provided in the papers [Li-S], [L-S1], and [Br1]. Free boundary problems from the viewpoint of variational inequalities are discussed in [L-S1], [K3], [K4], and [Ba1]. We shall cite more recent work in the course of our discussion. The first four sections of this paper concern some familiar problems whereas the last three are devoted to very recent considerations in the study of free boundaries. Our understanding of this topic is still rudimentary.

The methods we discuss may be applied to treat problems of physical interest. Among these are questions of fluid mechanics ([Br-S2], [BR-D]), hydraulics ([Ba1], [Ba2], [S4], [C1], [F-J1], [F-J2], [A1]), elasticity ([Ti], [Br2], [Br-S1], [C-N3]), plasma physics ([Te2], [Be-Br], [P], [K-Sp]), and hydrodynamics [Fr-Ber]. More complete references are given in the text.

Stopping time and impulse control problems lead to the notion of quasivariational inequalities. Spatial limitations have prevented the inclusion of this topic here. We refer to [Ben-Li1], [Ben-Li2], [F-Ben], [F4], [An-F]. We have also omitted a discussion of the theory of thin obstacles ([Fre2], [Fre3], [Giu]).

The author wishes to acknowledge his indebtedness to Professor Nestor Riviere for his vigorous interest in this work.

\section{Contents}

1. The obstacle problem

2. Regularity of the solution

3. The one-phase Stefan problem

4. Free boundary problems: the nature of the coincidence set

5. Recent directions in free boundary problems

6. The confined plasma

7. A theorem of Hans Lewy

1. The obstacle problem. We begin with an informal description of what has been generally referred to as the "obstacle problem." Let $\Omega \subset \mathbf{R}^{n}$ be open, connected with smooth boundary $\partial \Omega$ and let $\psi \in C^{2}(\bar{\Omega})$ satisfy

An expanded version of an address presented to the annual Meeting of the American Mathematical Society in St. Louis, Missouri on January 27, 1977; received by the editors May 5, 1977.

AMS (MOS) subject classifications (1970). Primary 35-XX, 49-XX.

${ }^{1}$ The preparation of this paper was partially supported by NSF grant MPS 75-06489. 


$$
\max _{\Omega} \psi>0 \text { and } \psi<0 \text { on } \partial \Omega .
$$

Consider a function $u(x)$ satisfying

(1.1) $\frac{1}{2} \int_{\Omega} u_{x}^{2} d x=\inf \left\{\frac{1}{2} \int_{\Omega} v_{x}^{2} d x: v \in C^{1}(\bar{\Omega}), v>\psi\right.$ in $\Omega, v=0$ on $\left.\Omega\right\}$.

Here $u_{x}=\left(u_{x_{1}}, \ldots, u_{x_{n}}\right)$.

Suppose, for the moment, that $u$ exists and $u \in C^{1}(\Omega)$.

The set $\Omega$ may be divided into the two subsets

$$
\begin{aligned}
I & =I(u)=\{x \in \Omega: u(x)=\psi(x)\}, \text { closed, and } \\
\Omega-I & =\{x \in \Omega: u(x)>\psi(x)\}, \text { open. }
\end{aligned}
$$

The set $I$ is called the coincidence set of $u$. One anticipates that

$$
\Delta u=0 \text { in } \Omega-I \text {, }
$$

whereas, at least formally,

$$
\Delta u=\Delta \psi \text { in } I .
$$

Notice this immediately suggests that the second derivatives of the solution may not be continuous in $\Omega$. In addition, $u-\psi$ attains its minimum value zero at any point of $I$; hence,

$$
u_{x_{i}}=\psi_{x_{i}} \text { on } I, \quad 1<i<n .
$$

In this fashion we may regard $u$ as the solution of a Cauchy problem

$$
\begin{array}{rlrl}
\Delta u & =0 & & \text { in } \Omega-I, \\
u & =\psi, & \\
u_{x_{i}} & =\psi_{x_{1}} \quad & & \text { on } \partial I .
\end{array}
$$

Since the Cauchy problem for $\Delta$ is not well posed even for a smooth initial surface $\partial I$, the mere existence of a solution suggests restrictive conditions on $\partial I$. The set $\partial I$ is what we call a "free boundary".

The set of functions

$$
\mathbf{K}_{\psi}=\left\{v \in C^{1}(\Omega): v \geqslant \psi \text { in } \Omega, v=0 \text { on } \partial \Omega\right\}
$$

is convex, so that $u+t(v-u) \in \mathbf{K}_{\psi}$ whenever $u, v \in \mathbf{K}_{\psi}$ and $0<t<1$. If $u$ has minimum Dirichlet integral in $\mathbf{K}_{\psi}$ then

$$
F(t)=\frac{1}{2} \int_{\Omega}\left(u_{x}+t(v-u)_{x}\right)^{2} d x, \quad 0<t<1,
$$

has a minimum at $t=0$, whence

$$
0<F^{\prime}(0)=\int_{\Omega} u_{x_{1}}(v-u)_{x_{1}} d x .
$$

Summation over repeated indices is understood. We express this condition as

$$
u \in \mathbf{K}_{\psi}: \int_{\mathbf{\Omega}} u_{x_{i}}(v-u)_{x_{i}} d x>0 \text { for } v \in \mathbf{K}_{\psi} .
$$

It is, by and large, the way in which variational inequalities are formulated. The convexity of the function $\frac{1}{2} p^{2}, p=\left(p_{1}, \ldots, p_{n}\right)$, easily leads to the conclusion that (1.5) implies (1.1). 
The analogy between (1.1) and (1.5) and the variational solution of an elliptic boundary value problem is more than casual. It would be legitimate to write that the theory of variational inequalities has as its model the variational theory of boundary value problems, but differs from this theory in that the competing functions belong to a convex set instead of an affine space. Just as in the theory of boundary value problems, the use of a minimization principle akin to Dirichlet's Principle to achieve the existence of $u$ entails widening the notion of solution, in the present case to functions in the Sobolev space

$$
v \in H_{0}^{1}(\Omega)=\left\{v \in L^{2}(\Omega): v_{x_{i}} \in L^{2}(\Omega), 1<i<n, v=0 \text { on } \partial \Omega\right\} .
$$

Recall that $H_{0}^{1}(\Omega)$ is a Hilbert space.

Henceforth we consider an enlarged convex set, still called $\mathbf{K}_{\psi}$,

$$
\mathbf{K}_{\psi}=\left\{v \in H_{0}^{1}(\Omega): v \geqslant \psi \text { in } \Omega\right\},
$$

where the notion of $>$ is defined appropriately [S1].

The existence of the solution $u$ of (1.5) now follows by direct methods. Indeed, as one suspects,

$$
u=P \psi, \quad \text { where } P: H_{0}^{1}(\Omega) \rightarrow \mathrm{K}_{\psi}
$$

is the projection operator from $H_{0}^{1}(\Omega)$ onto the closed convex set $\mathbf{K}_{\psi}$.

A smooth function $w$ is superharmonic in $\Omega$ provided that $-\Delta w>0$ in $\Omega$. We say that $w \in H_{0}^{1}(\Omega)$ is superharmonic if

$$
\int_{\Omega} w_{x_{i}} \zeta_{x_{1}} d x \geqslant 0 \text { whenever } 0<\zeta \in H_{0}^{1}(\Omega) \text {. }
$$

With $u$ the solution of (1.5) and $\zeta \in H_{0}^{1}(\Omega), \zeta \geqslant 0$,

$$
v=u+\zeta \in \mathbf{K}_{\psi} \text {, }
$$

and hence $u$ is superharmonic in $\Omega$. It admits the characterization [L-S1] $u(x)=\inf \left\{v(x) \in H_{0}^{1}(\Omega) ; v\right.$ superharmonic, $v \geqslant \psi$ in $\Omega$, and $v \geqslant 0$ on $\left.\partial \Omega\right\}$.

From the Riesz Thoerem, we deduce the existence of a nonnegative measure $\mu$ such that

$$
\begin{aligned}
u(x) & =\int_{\Omega} G(x, y) d \mu(y), \quad x \in \Omega, \\
\operatorname{supp} \mu & \subset I,
\end{aligned}
$$

where $G(x, y)$ denotes the Green's function of the Dirichlet problem for $-\Delta$ in $\Omega$. This framework for variational inequalities which bears a strong resemblence to potential theory is developed in Lions and Stampacchia [Li-S] and Lewy and Stampacchia [L-S1].

Likewise, it is possible to prove old facts about potential theory using variational inequalities. As an illustration, G. Stampacchia [S-5] has given a new and very simple proof that the minimum of two superharmonics is superharmonic which remains valid in the context of divergence form second order elliptic operators with bounded measurable coefficients.

The question of whether or not $u \in C^{1}(\Omega)$ has become a question about 
the smoothness of the solution of (1.5). Since we already know that $u \notin$ $C^{2}(\Omega)$, this regularity theory will have to be especially invented for variational inequalities.

A second variational inequality we would like to describe briefly concerns the function of least area in the convex set

$$
\mathbf{K}_{\psi}^{\prime}=\{v \text { lipschitz in } \bar{\Omega}: v \geqslant \psi \text { in } \Omega, v=0 \text { on } \partial \Omega\} .
$$

The question is to find

$$
u \in \mathbf{K}_{\psi}^{\prime}: \int_{\Omega} \frac{u_{x_{i}}}{\sqrt{1+u_{x}^{2}}}(v-u)_{x_{i}} d x \geqslant 0 \text { for all } v \in \mathbf{K}_{\psi}^{\prime} .
$$

All the difficulties inherent in the lack of coerciveness of the pairing determined by the minimal surface operator,

$$
\langle A u, \zeta\rangle=\int_{\Omega} \frac{u_{x_{i}}}{\sqrt{1+u_{x}^{2}}} \zeta_{x_{i}} d x, \quad u, \zeta \text { lipschitz, }
$$

influence the existence considerations here. So one is led first to establish suitable $a$ priori estimates for $\left|u_{x}\right|$ in the manner required for the solution of the Dirichlet problem. In particular, the condition that the mean curvature of $\partial \Omega$ have the proper sign is imposed ([Ma1], [Ma2], [L-S2], [Gia-P], and also [J-Ser] for the Dirichlet problem).

This problem has also been considered from a parametric standpoint by $\mathbf{M}$. Miranda [Mi].

2. Regularity of the solution. Returning to our example (1.5), we give a short description of a method of penalization which leads to smoothness of $u$ [L-S1]. Formally, $u$ is the solution to the nonlinear boundary value problem

$$
\begin{aligned}
-\Delta u & =-\Delta \psi \theta(u-\psi) & & \text { in } \Omega, \\
u & =0 & & \text { on } \partial \Omega,
\end{aligned}
$$

where

$$
\theta(t)= \begin{cases}1, & t \leqslant 0 \\ 0, & t>0 .\end{cases}
$$

Recall here (1.2) and (1.3). Of course, it is difficult to give meaning to (2.1) since neither $u$ nor $\theta$ is continuous.

The method of penalization consists in replacing the variational inequality (1.5) by a family of nonlinear boundary value problems resembling (2.1) and then demonstrating that their solutions converge to the solution of the variational inequality. The principal difficulty lies in obtaining suitable $a$ priori estimates. Let $\theta_{\varepsilon}(t)$ be defined by

$$
\theta_{\varepsilon}(t)= \begin{cases}1 & t \leqslant 0, \\ 1-t / \varepsilon & 0 \leqslant t \leqslant \varepsilon, \quad 0<\varepsilon \leqslant 1, \\ 0 & t \geqslant \varepsilon,\end{cases}
$$

and set 


$$
u_{\varepsilon} \in H_{0}^{1}(\Omega):-\Delta u_{\varepsilon}=\max (-\Delta \psi, 0) \theta_{\varepsilon}\left(u_{\varepsilon}-\psi\right) \text { in } \Omega .
$$

One may show $u_{e}$ exists by the Schauder fixed point theorem, for example. One may further show that $u_{\varepsilon} \in \mathbf{K}_{\psi}$. The standard elliptic theory and the uniform boundedness of the right hand side of (2.2) insures that

$$
\left\|u_{\varepsilon}\right\|_{C^{1, \alpha}(\bar{\Omega})} \leqslant \text { const, } \quad 0 \leqslant \alpha<1 .
$$

Here $C^{1, \alpha}(\bar{\Omega})$ denotes the $C^{1}(\bar{\Omega})$ functions whose norm

$$
\|v\|_{C^{1, \alpha}}=\|v\|_{L^{\infty}(\Omega)}+\sup _{x, x^{\prime} \in \Omega} \frac{\left|v_{x}(x)-v_{x}\left(x^{\prime}\right)\right|}{\left|x-x^{\prime}\right|^{\alpha}}<\infty .
$$

It now follows, with the aid of the device of Minty, that

$$
u_{\varepsilon} \rightarrow u \text { in } C^{1, \alpha}(\bar{\Omega}) \text { for each } \alpha<1 .
$$

What appears to be a subtle refinement of this is the fact that $u_{x_{1} x_{j}} \in L^{\infty}(\Omega)$ ([Br-K], [Fre1], [Ge]). However this fact seems to be essential in the study of the coincidence set $I$. The proof depends on a theorem of Stampacchia [S-1].

3. The one-phase Stefan problem. The considerations of the previous section may be extended to the study of parabolic variational inequalities. A particular example is the one phase Stefan problem which is the description, typically, of the melting of a body of ice maintained at $0^{\circ} \mathrm{C}$ in contact with a region of water. The unknowns are the temperature distribution of the water and the ice-water interface. This temperature distribution is required to solve the heat equation and energy is conserved across the interface.

We begin with an abbreviated description of this problem in one space dimension. Let $0<x_{0}<R$ and $T>0$ be given. Our problem is

To find a curve $\Gamma: t=s(x), x \geqslant x_{0}$, and a function $\Theta(x, t)$ satisfying

$$
\begin{aligned}
s(x) & =0 & & \text { for } 0 \leqslant x \leqslant x_{0}, \\
-\Theta_{x x}+\Theta_{t} & =0 & & \text { in } \Omega=\{(x, t): s(x)<t<T\}, \\
\Theta & =0 & & \\
\Theta_{x} s^{\prime} & =-k & & \text { on } \Gamma, \\
\Theta(x, 0) & =h(x), & & 0<x<x_{0}, \\
\Theta(0, t) & =g(t), & & 0<t<T,
\end{aligned}
$$

where $k>0$ is the heat of fusion, $h(x) \geqslant 0$ is the initial temperature, and $g(t) \geqslant 0$ is the temperature of the boundary $t$.

Note that $\Theta(x, t)=\min \Theta=0$ for $(x, t) \in \Gamma$, by the maximum principle, so $\Theta_{x} \leqslant 0$ on $\Gamma$. Hence

$$
s^{\prime}(x) \geqslant 0, \quad x_{0}<x<R,
$$

so the curve $\Gamma$ is monotone. This is the property of the one phase problem which readily permits its formulation as a variational inequality. It consists of introducing a new dependent variable by means of integration of the old one. Let us describe this way of formulating the problem since it is also useful in the study of filtration (eq. [Ba1]), fluid dynamics [Br-S2], and potential theory [K-S1]. 
Suppose now that $\Theta$ and $\Gamma$ exist and set $\Theta=0$ in the complement of $\Omega$. Following Duvaut [D], set $Q=(0, R) \times(0, T)$ and define

$$
\begin{gathered}
u(x, t)=\int_{0}^{t} \Theta(x, \tau) d \tau, \quad(x, t) \in Q, \\
f(x)=\left\{\begin{array}{cc}
h(x), & 0<x<x_{0}, \\
-k, & x_{0}<x<R,
\end{array}\right. \\
\psi(t)=\int_{0}^{t} g(\tau) d \tau, \quad 0<t<T, \quad \text { and } \\
\mathbf{K}=\left\{v \in L^{\infty}(Q): v \geqslant 0 \text { a.e. in } Q\right\} .
\end{gathered}
$$

It is no loss in generality to assume $x<R$ for $(x, t) \in \Gamma$. We now compute the equation satisfied by $u(x, t)$ in $Q$. Suppose that $u(x, t)>0$. Then either $0<x<x_{0}$ and $t>0$ or $x_{0}<x<R$ and $t>s(x)$. Consider the second possibility, in which case

$$
u(x, t)=\int_{s(x)}^{t} \Theta(x, \tau) d \tau .
$$

Since $\Theta(x, s(x))=0$,

$$
u_{x}(x, t)=\int_{s(x)}^{t} \Theta_{x}(x, \tau) d \tau
$$

Now

$$
\begin{aligned}
u_{x x}(x, t) & =\int_{s(x)}^{t} \Theta_{x x}(x, \tau) d \tau-s^{\prime}(x) \Theta_{x}(x, s(x)) \\
& =\int_{s(x)}^{t} \Theta_{\tau}(x, \tau) d \tau+k=\Theta(x, t)+k=u_{t}(x, t)+k .
\end{aligned}
$$

In the same manner, when $0<x<x_{0}, t>0$ we find that

$$
u_{x x}(x, t)=\int_{0}^{t} \Theta_{\tau}(x, \tau) d \tau=u_{t}(x, t)-h(x) .
$$

Consequently we may divide $Q$ into the two sets defined by the conditions

$$
\left\{\begin{aligned}
\left(-u_{x x}+u_{t}\right)(v-u)-f(v-u) & =0 \\
u & >0
\end{aligned} \text { for } v \in \mathbf{K},\right.
$$

and

$$
\left\{\begin{aligned}
\left(-u_{x x}+u_{t}\right)(v-u)-f(v-u)=+k v & >0 \\
u & =0 .
\end{aligned} \text { for } v \in \mathbf{K}\right.
$$

Hence it is always the case that

$u \in \mathbf{K}$ and $\left(-u_{x x}+u_{t}\right)(v-u) \geqslant f(v-u)$ in $Q$ for all $v \in \mathbf{K}$.

These considerations lead to the parabolic variational inequality

$$
\left(-u_{x x}+u_{t}\right)(v-u)>f(v-u) \text { ae. in } Q \text { for all } v \in \mathbf{K} \text {, }
$$




$$
\begin{array}{ll}
u=0, & t=0,0<x<R, \\
u=\psi, & x=0,0<t<T, \\
u=0, & x=R, 0<t<T .
\end{array}
$$

This is analogous to (1.5) after an integration by parts. It is clear that $u \in K$ and that $\Omega=\{(x, t): u(x, t)>0\}$.

Without formulating the classical problem (cf., e.g., [F2] or [F-K]), we present the variational inequality associated to the Stefan problem for arbitrary dimension. We restrict our attention to a special case.

Suppose given an "annular" domain $G \subset \mathbf{R}^{n}$ whose boundary consists of $\partial B_{\delta}=\{x:|x|=\delta\}$, some $\delta>0$, and a smooth manifold $\Gamma_{0}$ with $\partial B_{\delta}$ lying inside $\Gamma_{0}$. Let $B$ denote a large ball with center at the origin containing $G$ and set $D=B-\bar{B}_{\delta}, Q=D \times(0, T)$ for a $T>0$. Let $k>0, h(x)$ be a smooth positive function in $G$, and $\psi(x, t)$ be a smooth positive function in $\partial B_{\delta} \times$ $(0, T)$. For the $n$-dimensional variational inequality, we are asked

To find $u(x, t) \in \mathbf{K}=\left\{v \in L^{\infty}(Q): v \geqslant 0\right.$ in $\left.Q\right\}$ such that $u_{x_{1}, x}, u_{t} \in$ $L^{2}(Q)$ and

$$
\begin{array}{rlrl}
\left(-\Delta u+u_{t}\right)(v-u) & >f(v-u) & \text { a.e. in } Q \text { for } v \in \mathbf{K}, \\
u(x, 0) & =0, & x \in D, \\
u(x, t) & =\psi(x, t), & & (x, t) \in \partial B_{\delta} \times(0, T), \\
u(x, t) & =0, & & |x|=R, 0<t<T .
\end{array}
$$

where

$$
f(x)= \begin{cases}h(x), & x \in G \\ -k, & x \in D-G\end{cases}
$$

as before.

Again we may inquire about the smoothness of the solution [F-K] and, indeed, in what sense $\Theta=u_{t}$ and $\Gamma=\partial \Omega \cap Q, \Omega=\{(x, t): u(x, t)>0\}$, comprise a solution to the classical Stefan problem. We discuss this in \$5. A general discussion of parabolic free boundary problems may be found in [Mag].

4. Free boundary problems: the nature of the coincidence set. This section will be devoted to a brief description of the coincidence set of (1.5) in the geometrically simplest cases. As we have mentioned in (1.4), this form of free boundary has a connection with the Cauchy problem for an elliptic equation. Not all free boundaries occur in this manner. For example, the classical free boundary problems associated with steady fluid motion, which arise from Bernoulli's Law, admit a different formulation. For an introduction to those considerations we refer to the book of P. Garabedian [G, Chapters 14, 15].

Suppose that

$\Omega \subset \mathbf{R}^{2}$ is strictly convex with smooth boundary $\partial \Omega$ and $\psi \in$ $C^{2}(\bar{\Omega})$ is a strictly concave obstacle with the properties $\max _{\Omega} \psi>0$ and $\psi<0$ on $\partial \Omega$.

Here is a summary of our knowledge about the coincidence set:

Let $u$ be the solution of (1.5), assume (4.1), and let $I=I(u)$ denote its set of 


\section{coincidence. Then}

(i) if $\psi$ is real analytic, $\partial I$ is an analytic Jordan curve,

(ii) if $\psi \in C^{2, \alpha}(\Omega), \partial I$ is a $C^{1, \alpha}$ Jordan curve, $0<\alpha<1$,

(iii) if $\psi \in C^{m, \alpha}(\Omega), \partial I$ is a $C^{m-1, \alpha}$ Jordan curve, $m \geqslant 2,0<\alpha<1$.

The conclusion (i) is due to H. Lewy and G. Stampacchia [L-S1], (ii) may be found in [K4], and (iii) in L. Caffarelli and N. Riviere [CR1]. The work [CR1] is considerably more general and contains an extensive characterization of $I$ without any geometric assumptions about $\Omega$ and $\psi$.

We might observe that if we assume only that $\psi \in C^{2}(\Omega)$, we may conclude, under the hypothesis (4.1), that $\partial I$ is a Jordan curve of measure zero. In fact, it is a quasi-conformal circle and thus admits a Hölder continuous parameterization ([K2], [K3]).

Naively, one may suspect $I$ to be convex. Let us dispell any hopes about this. If $\psi$ is the graph of a polyhedron, there is a Lipschitz solution to the variational inequality (1.5) [L-S2]. The graph of the solution, has contact with either an entire open face or with none of it ([Mu-S], [K1]), according to a special maximum principle. So the coincidence set consists of the projection in $\Omega$ of faces, subsets of edges, and vertices and may well be only edges and vertices. It is easy to find a sequence $\psi_{\varepsilon} \rightarrow \psi$ as $\varepsilon \rightarrow 0$ of $C^{\infty}$ strictly concave $\psi_{\varepsilon}$ whose coincidence sets $I_{\varepsilon}$ "converge" to $I$.

So, for example, if $\psi$ is a tetrahedron, the coincidence set of the solution to (1.5) has the shape of the letter $Y$. It cannot contain a face, for in that case, the superharmonic $u$ would attain an interior minimum. It follows that there are smooth concave obstacles for which the coincidence set is not convex. But is it always star-shaped? To carry this idea one step further, consider a tent-shaped polyhedron whose edges project to the configuration

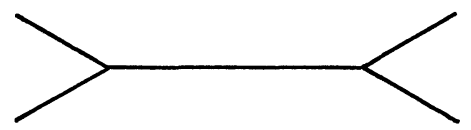

This leads to the discovery of a smooth obstacle with coincidence set which is not even star-shaped.

If the boundary conditions on $\partial \Omega$ are not homogeneous, then $\partial I$ may also admit cusps. David Schaeffer [Sc1] has given examples of this and also of much more bizarre situations (cf. also [KN1]).

We want to suggest the reasoning which leads to the conclusion (i) and the difficulties encountered in adapting it to (ii) and other questions. Suppose that $\omega$ is a simply connected bounded domain in the $z=x_{1}+i x_{2}$ plane with $\Gamma \subset \partial \omega$ a smooth Jordan arc. Consider functions $u, \psi$ satisfying

$u \in C^{1}(\omega \cup \Gamma), \psi$ real analytic in a neighborhood of $\omega \cup \Gamma$,

$\Delta u=0$ in $\omega$,

(4.2) $\left\{\begin{array}{c}u=\psi \\ u_{x_{j}}=\psi_{x_{j}}\end{array}\right.$ on $\Gamma, k=1,2$,

$\Delta \psi \neq 0$ in $\overline{\omega \cup \Gamma}$. 
Suppose that $z=0 \in \Gamma$. The complex gradient $f(z)=u_{x_{1}}-i u_{x_{2}}$ is holomorphic in $\omega$ and has continuous boundary values $F(z, \bar{z})=\psi_{x_{1}}-i \psi_{x_{2}}$ for $z \in \Gamma$. In a neighborhood of $z=0$, consider the equation

$$
f(z)=F\left(z, z^{*}(z)\right)
$$

keeping in mind that $z^{*}(z)=\bar{z}$ is a solution of (4.3) whenever $z \in \Gamma$. Since

$$
\left.\frac{\partial}{\partial z^{*}} F\left(z, z^{*}\right)\right|_{\left(z, z^{*}\right)=(0,0)}=\frac{1}{2} \Delta \psi(0) \neq 0,
$$

we infer the existence of a solution $z^{*}=\zeta(z, f)$ of $(4.3)$ holomorphic in $(z, f)$ near $(0, f(0))$. In particular

$$
z^{*}(z)=\zeta(z, f(z)), \quad z \in \omega,|z| \text { small, }
$$

is holomorphic in $z$. By the uniqueness statement of the implicit function theorem

$$
z^{*}(z)=\bar{z}, \quad z \in \Gamma,|z| \text { small. }
$$

At this stage, we have represented $\bar{z}$ on $\Gamma$ as the boundary value of a function holomorphic in $\omega$. This implies that $\Gamma$ is analytic by the Schwarz Reflection Principle. To verify this conclusion, let

$$
\begin{aligned}
& \varphi: G \rightarrow \omega, \quad G=\left\{t=t_{1}+i t_{2}:|t|<1, \operatorname{Im} t>0\right\}, \\
& \varphi(0)=0
\end{aligned}
$$

be a conformal mapping of $G$ onto $\omega$ which transforms the real segment $(-1,1)$ onto $\Gamma$. We know that $\varphi \in C(G \cup(-1,1))$. Define

$$
\Phi(t)= \begin{cases}\frac{\varphi(t),}{z^{*}(\varphi(\bar{t}))}, & \operatorname{Im} t \geqslant 0,|t|<1, \\ \operatorname{Im} t|t|<1,\end{cases}
$$

a holomorphic function in $G \cup\{t:|t|<1, \operatorname{Im} t<0\}$. When $t$ is real, (4.4) insures that $\Phi$ is continuous. By Morera's Theorem, $\Phi$ is holomorphic in a neighborhood of $t=0$. Consequently,

$$
t \rightarrow \Phi(t), \quad t \text { real, }|t| \text { small, }
$$

exhibits an analytic parametrization of a portion of $\Gamma$.

It was observed in [L-S1] that the extension (4.5) is valid if $\Gamma$ is known only to be a continuum. This is the idea of the proof of (i). It fails, evidently, when $\psi$ is not analytic. Indeed, the definition $z^{*}=\zeta(z, f)$ is suspect in this situation.

From a slightly different viewpoint, (4.5) offers an extension of $\varphi$ to a function $\Phi$ satisfying the homogeneous analytic equation $(\partial / \partial \bar{t}) \Phi=0$. In general, it is not possible to achieve an extension of this nature so an alternative idea has been devised which might be interpreted as a combination of classical potential theory and some ideas about Sobolev spaces, especially their trace classes (cf. [K3], [K4], and [K-S, Chapter V] for the simplest treatment).

An extension of the conformal mapping based on (4.3) is not always convenient. For example, in (1.7) the complex gradient is not holomorphic. Here the demonstration of analyticity of $\partial I$ relies on the resolution of a 
system of differential equations to extend analytically a conformal representation of the minimal surface which is the graph of $u$ in $\Omega-I$.

The idea of connecting an analytic function to its possible extension by means of the solution to a differential equation is due to Hans Lewy ([L2], [L3], [LA], [L1]). A particularly lucid description of his idea is presented in the paradigm [L1]. We shall return to [LA] in \$7.

5. Recent directions in free boundary problems. Here we intend to discuss some work in [K-N1] and some recent work of L. Caffarelli ([C1], [C2]). At the conclusion of this section we describe the smoothness properties of the ice-water interface in the Stefan problem. We begin from a formal point of view with the notions of hodograph and Legendre transformations. These have the effect of "straightening" the free boundary at the expense of introducing a highly nonlinear equation.

Suppose that $u$ is a solution of (1.5) with

$$
-\Delta \psi>0 \text { and analytic. }
$$

In addition, assume that

$$
\begin{aligned}
& \Gamma \subset \partial I \text { is a } C^{1} \text { hypersurface and } \\
& u_{i j} \in C(\Gamma \cup(\Omega-I)), 1<i, j<n,
\end{aligned}
$$

where $u_{i j}=u_{x_{i} x_{j}}$. We assume $0 \in \Gamma$ and that the inward normal to $\Omega-I$ at 0 is in the direction of the positive $x_{1}$-axis. Set $w=u-\psi$. Then, analogous to (1.4),

$$
\begin{array}{rlrl}
\Delta w & =a & & \text { in } \Omega-I, \\
w & =0 & \text { on } \Gamma, \\
w_{i} & =0, & 1 \leqslant i \leqslant n,
\end{array}
$$

where $a \equiv-\Delta \psi>0$ is analytic in a neighborhood of $x=0$. We introduce the change of variables

$$
\begin{aligned}
& y_{1}=-w_{1} \equiv w_{x_{1}}, \\
& y_{\alpha}=x_{\alpha}, \quad 2<\alpha<n,
\end{aligned}
$$

and the function

$$
v(y)=x_{1} y_{1}+w(x)
$$

We refer to (5.4) as a partial hodograph transformation and to $v$ in (5.5) as the Legendre transform of $w$. Note that (5.4) and (5.5) differ from the customary definitions by a change in sign.

It is easy to see that (5.4) is $1: 1$ near $x=0$. Indeed, since $w_{i}=0$ on $\Gamma$ and $(1,0, \ldots, 0)$ is the normal to $\Gamma$ at 0 ;

$$
w_{i \alpha}(0)=0, \quad 1<i \leqslant n, 2 \leqslant \alpha \leqslant n,
$$

so from (5.3), $w_{11}(0)=a(0)>0$. Hence $d y / d x(0)$ is nonsingular. Under the mapping (5.4), a neighborhood of $x=0$ in $\Omega-I$ is mapped onto a set $U \subset\left\{y: y_{1}>0\right\}$ and a neighborhood of $x=0$ in $\Gamma$ is mapped onto

$$
\Sigma \subset\left\{y: y_{1}=0\right\} \text {. }
$$

The property of the Legendre transform is that 


$$
\begin{aligned}
d v & =x_{1} d y_{1}+y_{1} d x_{1}+d w \\
& =x_{1} d y_{1}+\sum_{\alpha>1} w_{\alpha} d y_{\alpha}
\end{aligned}
$$

or

$$
\begin{aligned}
& v_{1}=x_{1}, \\
& v_{\alpha}=w_{\alpha}, \quad 2 \leqslant \alpha \leqslant n .
\end{aligned}
$$

We write $v_{i}$ for $v_{y i}$. In particular, a portion $\Gamma^{\prime}$ of $\Gamma$ admits the parametrization

$$
\Gamma^{\prime}: x_{1}=\frac{\partial v}{\partial y_{1}}\left(0, x_{2}, \ldots, x_{n}\right), \quad\left(0, x_{2}, \ldots, x_{n}\right) \in \Sigma .
$$

The smoothness of $\Gamma^{\prime}$ has become a question of that of $v$ in $U \cup \Sigma$. One easily computes from (5.3), setting $y^{\prime}=\left(y_{2}, \ldots, y_{n}\right)$, that

$$
\begin{aligned}
-\frac{1}{v_{11}}-\frac{1}{v_{11}} \sum_{\alpha>1} v_{1 \alpha}^{2}+\sum_{\alpha>1} v_{\alpha \alpha}-a\left(v_{1}, y^{\prime}\right) & =0 \text { in } U, \\
v & =0 \text { on } \Sigma .
\end{aligned}
$$

Note that $w_{11}=-1 / v_{11}<0$ in $U$.

The equation of (5.8) is elliptic and analytic near $y=0$ in $U$; hence, by a well-known theorem ([ADN1], [F1], [Mo]), $v(y)$ is analytic in $U \cup \Sigma$. Therefore $\Gamma^{\prime}$ is also analytic.

In summary, once (5.2) is assumed, the free boundary is analytic provided the obstacle $\psi$ is. Similarly, if $\psi \in C^{m, \alpha}(\Omega)$, then $\Gamma$ is of class $C^{m-1, \alpha}$, $0<\alpha<1$. This idea may be implemented in many other problems, including, incidentally, those of steady fluid motion mentioned in the beginning of $\$ 4$. Again we refer to [K-N1] for details.

To affirm the hypothesis (5.2), L. Caffarelli has developed a theory about the initial regularity of $\Gamma$. We describe the principal conclusion [C2], Theorem 3. Set $B_{\rho}(0)=\left\{x \in \mathbf{R}^{n}:|x|<\rho\right\}$.

Suppose that $0 \in \Gamma$ and

$$
\liminf _{\rho \rightarrow 0} \frac{\operatorname{meas}\left(B_{\rho}(0) \cap I\right)}{\operatorname{meas}\left(B_{\rho}(0)\right)}>0 .
$$

Then there is a neighborhood $B_{r}(0)$ such that $\Gamma \cap B_{r}(0)$ is a $C^{1}$ hypersurface and

$$
u_{i j} \in C\left((\Omega-I) \cup\left(\Gamma \cap B_{r}(0)\right)\right), \quad 1 \leqslant i, j \leqslant n .
$$

The proof entails a careful analysis of the geometry of $I$. A major step is to show that the points of $\partial I$ possessing positive density with respect to $I$ constitute an open subset of $\partial I$. We shall give an outline of the proof.

Given a bounded set $S \subset \mathbf{R}^{n}$, let us call min diam $S$ the infimum of the distances between pairs of parallel planes which enclose $S$. Given $x_{0} \in \partial I$, Caffarelli determines a continuous increasing function $\omega(\rho)$ with $\omega(0)=0$ such that either

$$
\min \operatorname{diam}\left(I \cap B_{\rho}\left(x_{0}\right)\right)<\omega(\rho) \rho
$$

for all small $\rho$ or if for some small $\rho_{0}>0, \min \operatorname{diam}\left(I \cap B_{\rho_{0}}\left(x_{0}\right)\right)>\omega\left(\rho_{0}\right) \rho_{0}$, 
then

$$
\min \operatorname{diam}\left(I \cap B_{\rho}\left(x_{0}\right)\right)>\omega\left(\rho_{0}\right) \rho \text { for all } \rho \leqslant \rho_{0} .
$$

By a geometric argument one may establish that $I \cap B_{\rho}(x)$ contains a ball of radius proportional to min $\operatorname{diam}\left(B_{\rho}(x) \cap I\right)$, at least for small $\rho$, so (5.9) implies that

$$
\frac{\text { meas } B_{\rho}\left(x_{0}\right) \cap I}{\text { meas } B_{\rho}\left(x_{0}\right)} \geqslant c>0 \text { for } \rho \leqslant \rho_{0} \text { and some } c>0 .
$$

With this in mind it is not difficult to prove that if a point of $\partial \Gamma$ has positive density with respect to $I$ then a neighborhood of it in $\partial I$ shares this property.

These considerations extend to the Stefan problem. For example, there are known conditions about the boundary temperature $\psi_{t}$ of (3.2) and the initial temperature $g$ of (3.3) which insure that $\Gamma$ is Lipschitz in the space variables for each fixed time [F-K, Theorem 5]. This is sufficient to apply Caffarelli's work [C2], cf. also [K-N2], to conclude that $\Gamma$ is a $C^{1}$ manifold and that the second derivatives $u_{i j}, u_{t j}, 1 \leqslant i, j \leqslant n$, are continuous in $\Omega \cup \Gamma$.

It is worth noting that the interface $\Gamma$ separating the ice and water regions at a given time $t$ depends only on the data prescribed through time $t$. Therefore $\Gamma$ cannot in general depend analytically on the time variable. However, it exhibits rather precise behavior: it varies analytically with the space variables and is in the second Gevrey class with respect to time. Moreover, in any time interval during which the contributed heat is analytic, the interface depends analytically on time. Both of these statements are proved in [K-N2], [K-N3]. In one space dimension, the analyticity of the free curve was shown by Avner Friedman [F3].

6. The confined plasma. Consider an axially symmetric toroidal vessel which contains an ionized gas, for example hydrogen, held in equilibrium by an externally applied magnetic field. We are asked to find the magnetic vector $B$ of the vessel a part of which is occupied by the gas while the rest is a vacuum. The magnetic vector satisfies a different relation in each region so in particular its determination locates the space occupied by the gas. For expository purposes, it is easier to suppose the gas contained in an infinite cylinder of constant cross-section $\Omega \subset \mathbf{R}$, where $\Omega$ is bounded and simply connected.

The magnetic vector in this configuration admits a stream function $u$, namely $B=\left(B_{1}, B_{2}, B_{3}\right)$ with $\left(B_{1}, B_{2}, 0\right)=\operatorname{curl}(0,0, u)=\left(u_{x_{2}},-u_{x_{1}}, 0\right)$ and $B_{3}=B_{3}(u)$.

In the simplest model, the problem reduces to this:

Given $\lambda>0, I>0$, find $u \in H^{1}(\Omega)$ satisfying

$$
\begin{aligned}
\Delta u+\lambda \min (u, 0) & =0 & & \text { in } \Omega, \\
u & =c>0 & & \text { on } \partial \Omega, \\
\int_{\partial \Omega} \frac{\partial u}{\partial \nu} d s & =I & &
\end{aligned}
$$

where $c>0$ is a constant to be determined; $\nu$ denotes the outward normal to $\Omega$. 
The set $\Omega_{p}=\{x: u(x)<0\}$ is the cross-section of the plasma region and $\Omega-\bar{\Omega}_{p}=\{x: u(x)>0\}$ is the cross-section of the vacuum region. We shall show that $\Gamma_{p}=\partial \Omega_{p}$ is an analytic Jordan curve [KNS] by transforming the question into one about an elliptic system with complementing boundary conditions [ADN2]. A similar problem with the same "free boundary" condition occurs in the theory of the hydrodynamical vortex [Fr-Ber].

A description of the physical basis for this model may be found in $\mathbf{R}$. Temam [Te1] or Shafranov [Sh]. Solutions to (6.1) have been found by Berestyeki-Brezis [Be-Br], Puel [P], and Temam ([Te2], [Te3]). The solution is not generally unique, cf. D. Schaeffer [Sc2], but the works cited above contain appropriate uniqueness statements.

In [Te3], the solution is found to be the function $u$ miminizing

$$
E(v)=\frac{1}{2} \int_{\Omega} v_{x}^{2} d x-\frac{\lambda}{2} \int_{\Omega} \min (v, 0)^{2} d x-\left.I v\right|_{\partial \Omega}
$$

among all functions $v$ satisfying

$$
\int_{\Omega}|\min (v, 0)| d x=\frac{I}{\lambda},\left.\quad v\right|_{\partial \Omega} \quad \text { is constant. }
$$

Note that by a result of Stampacchia [S1], any solution $u$ of (6.1) satisfies $u \in C^{2, \alpha}(\Omega), 0<\alpha<1$.

It is possible to show that for the solution of (6.1) obtained via (6.2), (6.3) [K-Sp], $\Gamma_{p}=\partial \Omega_{p}$ is a $C^{2}$-Jordan curve. In particular, $\Omega_{p}$ is connected.

To explore the question of the analyticity of $\Gamma_{p}$, we consider a local situation. Suppose that $\Gamma$ is a $C^{1}$-Jordan arc contained in $B=\left\{x \in \mathbf{R}^{2}\right.$ : $|x|<1\}$ joining $(-1,0)$ to $(1,0)$ passing through $(0,0)$. Let $U_{+}$and $U_{-}$ denote the components of $B-\Gamma$ containing $(0,1)$ and $(0,-1)$ respectively. Suppose now that

$$
\begin{aligned}
& u \in C^{2}(B): \quad \Delta u=0 \text { in } U_{+}, \\
& \Delta u+\lambda u=0 \text { in } U_{-} \text {, } \\
& \left\{\begin{aligned}
u & =0 \\
\partial u / \partial \nu & \neq 0
\end{aligned} \text { on } \Gamma,\right.
\end{aligned}
$$

where $\nu$ is a normal direction to $\Gamma$.

We may assume that $u>0$ in $U_{+}$and $u<0$ in $U_{-}$and that $(0,1)$ is a normal direction to $\Gamma$ at $(0,0)$.

Consider the transformation

$$
\begin{aligned}
& y_{1}=x_{1}, \\
& y_{2}=u(x), \quad|x| \text { small, }
\end{aligned}
$$

and the new dependent variable

$$
v(y)=x_{2}
$$

This mapping is $1: 1$ near $x=(0,0)$ because $\partial u / \partial \nu(x) \neq 0$ on $\Gamma$. A subarc of $\Gamma$ is mapped onto a segment $\sigma$ of the $y_{1}$-axis and neighborhoods $U_{ \pm} \cap N$ are 
mapped into domains $G_{ \pm}$contained in $\left\{y_{2}>0\right\}$ and $\left\{y_{2}<0\right\}$ respectively. Say $G_{+}=\left\{y:|y|<\varepsilon, y_{2}>0\right\}, G_{-}=\left\{y:|y|<\varepsilon, y_{2}<0\right\}$, and $\sigma=$ $\left\{\left(y_{1}, 0\right):\left|y_{1}\right|<\varepsilon\right\}$.

It is easy to verify that

$$
\Delta u=F\left(v_{y y}, v_{y}\right)=-\frac{v_{11}}{v_{2}}+2 \frac{v_{1}}{v_{2}^{2}} v_{12}-\frac{\left(1+v_{1}^{2}\right)}{v_{2}^{3}} v_{22}
$$

whence from (6.4),

$$
\begin{aligned}
F\left(v_{y y}, v_{y}\right) & =0 \text { in } G_{+}, \\
F\left(v_{y y}, v_{y}\right)+\lambda y_{2} & =0 \text { in } G_{-},
\end{aligned} \quad v \in C^{1}\left(G_{+} \cup \sigma \cup G_{-}\right) .
$$

Now reflect $v$ in $G_{-}$into $G_{+}$defining

$$
w(y)=v\left(y_{1},-y_{2}\right) \text { for } y \in G_{+} .
$$

After a brief calculation we are led to the elliptic boundary value problem

$$
\begin{aligned}
& F\left(v_{y y}, v_{y}\right)=0 \\
& \begin{aligned}
F\left(w_{y y}, w_{y}\right)+\lambda y_{2} & =0 \\
v-w & =0
\end{aligned} \\
& v_{2}+w_{2}=0 \\
& \text { in } G_{+} \text {, }
\end{aligned}
$$

This problem is coercive, or complementing [ADN2], so $v(y)$ and $w(y)$ are analytic in $G_{+} \cup \sigma[M o$, p. 271]. From (6.6) we see that a neighborhood of $(0,0)$ in $\Gamma$ is analytic.

The two phase Stefan problem is also amenable to this formulation [K-N-S].

7. A theorem of Hans Lewy. In his work [LA], H. Lewy observed that solutions of the Laplace equation and its associated eigenvalue problem are co-extensive on analytic arcs. More precisely, let $G=\left\{x \in \mathbf{R}^{2}:|x|<1\right.$, $\left.x_{2}>0\right\}, \sigma=\left\{x: x_{2}=0,\left|x_{1}\right|<1\right\}$, and suppose $u, v \in C^{1}(G \cup \sigma)$ satisfy

$$
\begin{aligned}
& \Delta u=0 \text { in } G, \\
& \Delta v+\lambda v=0 \\
& u-v=0 \\
& u_{2}-v_{2}=0
\end{aligned} \quad \text { on } \sigma,
$$

where $\lambda \neq 0$ is a number.

Then $u, v$ are analytically extensible into a neighborhood of $x=0$. Lewy's proof uses the ideas mentioned in \$4. The result was generalized to higher dimensions by C. Kahane [Ka]. Obviously, (7.1) is not coercive. 
We begin with an elementary proof of Lewy's result motivated by our present ideas [KNS]. Set

$$
w=u-v, \quad z=u+v .
$$

Then

$$
\begin{gathered}
z=\frac{2}{\lambda}\left(\Delta w+\frac{\lambda}{2} w\right) \text { and } \\
\Delta^{2} w+\lambda \Delta w=0 \\
\begin{cases}w=0 \\
w_{2}=0\end{cases}
\end{gathered}
$$

The boundary conditions on $\sigma$ for the equation in (7.3) are analytic and complementing [A-D-N1]. Hence $w$ is analytic in $G \cup \sigma$. The complementing condition in this instance means that the boundary conditions of (7.3), when suitably extended to all of $\partial G$, and the fourth order elliptic equation constitute a well posed boundary value problem. By (7.2), $z$ is also analytic. Consequently $u$ and $v$ are analytic near $G \cup \sigma$. Notice the importance of the fact that $\lambda \neq 0$. This was not needed in the plasma problem (6.4).

We introduce a free boundary problem associated to the system (7.1). Let $\boldsymbol{\Omega} \subset \mathbf{R}^{n}$ be a simply connected domain whose boundary contains a smooth hypersurface $\Gamma$. Suppose that $u^{1}, u^{2} \in C^{3}(\Omega \cup \Gamma)$ satisfy

$$
\begin{aligned}
& \Delta u^{1}=0 \\
& \Delta u^{2}+u^{2}=0 \\
&\left\{\begin{array}{ll}
u^{1} & =u^{2}=0 \\
u_{\nu}^{1} & =u_{\nu}^{2} \neq 0
\end{array} \quad \text { in } \Omega,\right.
\end{aligned}
$$

where $\nu$ denotes the normal direction on $\Gamma$.

We shall show that $\Gamma$ is analytic [KNS]. With some imagination the equations (7.2), (7.3) may be regarded as a fourth order elliptic system. Again we shall pass to a fourth order problem, on this occasion a true system.

The conditions fulfilled by $u^{i}$ on $\Gamma$ are, in essence, necessary. In particular, the conclusion fails if $\partial u^{i}(x) / \partial \nu=0$ for some $x \in \Gamma$ or if we assume only that $u^{1}=u^{2}$ on $\Gamma$.

For the proof, set

$$
w=u^{1}-u^{2} \text { and } z=u^{1}+u^{2}
$$

or

$$
u^{1}=\frac{1}{2}(w+z), \quad u^{2}=-\frac{1}{2}(w-z) .
$$

From these relations we compute that

$$
z=2 \Delta w+w \text { in } \Omega
$$




$$
\Delta^{2} w+\Delta w=0 \text { in } \Omega
$$

$$
w=0, \quad w_{\nu}=0, \quad w_{\nu \nu}=0, \quad w_{v v} \neq 0 \text { on } \Gamma .
$$

The last condition follows from the fact that

$$
\frac{\partial z}{\partial \nu}=2 \frac{\partial u^{i}}{\partial \nu} \neq 0
$$

and (7.6).

Suppose that $0 \in \Gamma$ and that the $x_{n}$-axis is in the normal direction to $\Omega$ at $0 \in \Gamma$. Introduce new independent variables

$$
y=\left(x^{\prime},-w_{n n}\right) \quad \text { near } x=0, x^{\prime}=\left(x_{1}, \ldots, x_{n-1}\right)
$$

and dependent variables

$$
\begin{aligned}
& v^{1}(y)=x_{n} y_{n}+w_{n}(x), \\
& v^{2}(y)=\sum_{\alpha \leqslant n-1} w_{\alpha \alpha}(x) .
\end{aligned}
$$

In view of (7.8), the mapping (7.9) carries a neighborhood of 0 in $\Omega$ onto a neighborhood $U$ of $y=0$ in a halfspace, say $U \subset\left\{y: y_{n}>0\right\}$ and an appropriate portion of $\Gamma$ onto $\Sigma=\partial U \cap\left\{y_{n}=0\right\}$.

We determine a relationship between $v^{1}$ and $v^{2}$ from (7.7). We agree that the subscripts of the $v^{i}$ refer to differentiation with respect to $y$ and those of $w$ refer to differentiation with respect to $x$. Now $v^{1}$ is the Legendre transform of $w_{n}$ with respect to (7.9), so

In addition

$$
\begin{aligned}
& v_{\alpha}^{1}=w_{\alpha n}, \quad 1 \leqslant \alpha \leqslant n-1, \\
& v_{n}^{1}=x_{n} .
\end{aligned}
$$

$$
\begin{gathered}
w_{n n n}=-\frac{1}{v_{n n}^{1}}, \quad w_{n n n n}=\frac{v_{n n n}^{1}}{\left(v_{n n}^{1}\right)^{3}}, \\
w_{n n \alpha \alpha}=\left(\frac{v_{n \alpha}^{1}}{v_{n n}^{1}}\right)_{\alpha}-\frac{v_{n \alpha}^{1}}{v_{n n}^{1}}\left(\frac{V_{n \alpha}^{1}}{v_{n n}^{1}}\right)_{n}, \quad 1 \leqslant \alpha \leqslant n-1,
\end{gathered}
$$

and

$$
\begin{aligned}
\sum_{\alpha<n} w_{\alpha \alpha \beta}=v_{\beta}^{2}-\frac{v_{n \beta}^{1}}{v_{n n}^{1}} v_{n}^{2}, \quad 1 \leqslant \beta \leqslant n-1, \\
\sum_{\alpha<n} w_{\alpha \alpha \beta \beta}=v_{\beta \beta}^{2}-2 \frac{v_{n \beta}^{1}}{v_{n n}^{1}} v_{n \beta}^{2}+\left[\frac{v_{n \beta}^{1}}{v_{n n}^{1}}\right]^{2} v_{n n}^{2} \\
-\left[\frac{v_{n \beta}^{1}}{v_{n n}^{1}}\right]_{\beta}^{2}+\left[\frac{v_{n \beta}^{1}}{v_{n n}^{1}}\right]_{n}\left[\frac{v_{n \beta}^{1}}{v_{n n}^{1}}\right] v_{n}^{2} .
\end{aligned}
$$


Let us write

$$
\begin{aligned}
& 0=\Delta^{2} w+\Delta w, \\
& 0=w_{n n n n}+2 \sum_{\alpha<n} w_{\alpha \alpha n n}+\sum_{\alpha, \beta<n} w_{\alpha \alpha \beta \beta}+w_{n n}+\sum_{\alpha<n} w_{\alpha \alpha},
\end{aligned}
$$

consequently

$$
\begin{gathered}
F_{1}\left(v^{1}, v^{2}, y\right)=0 \text { in } U, \text { where } \\
F_{1}\left(v^{1}, v^{2}, y\right)=\frac{v_{n n}^{1}}{\left(v_{n n}^{1}\right)^{3}}+2 \sum_{\alpha<n}\left\{\left(\frac{v_{n \alpha}^{1}}{v_{n n}^{1}}\right)_{\alpha}-\frac{v_{n \alpha}^{1}}{v_{n n}^{1}}\left(\frac{v_{n \alpha}^{1}}{v_{n n}^{1}}\right)_{n}\right\} \\
+\sum_{\alpha, \beta<n}\left\{v_{\beta \beta}^{2}-2 \frac{v_{n \beta}^{1}}{v_{n n}^{1}} v_{n \beta}^{2}+\left[\frac{v_{n \beta}^{1}}{v_{n n}^{1}}\right]^{2} v_{n n}^{2}-\left[\frac{v_{n \beta}^{1}}{v_{n n}^{1}}\right]_{\beta}^{v_{n}^{2}}\right. \\
\left.+\left[\frac{v_{n \beta}^{1}}{v_{n n}^{1}}\right]_{n}\left[\frac{v_{n \beta}^{1}}{v_{n n}^{1}}\right] v_{n}^{2}\right\}+v^{2}-y_{n} .
\end{gathered}
$$

The second equation is obtained by differentiating $v^{2}$ in two ways:

$$
\sum_{\alpha<n} w_{\alpha \alpha n}=\frac{v_{n}^{2}}{v_{n n}^{1}} \text { and } \sum_{\alpha<n} w_{\alpha n \alpha}=\sum_{\alpha<n}\left[v_{\alpha \alpha}^{1}-\frac{\left(v_{\alpha n}^{1}\right)^{2}}{v_{n n}^{1}}\right] .
$$

So

$$
F_{2}\left(v^{1}, v^{2}\right)=v_{n}^{2}-\sum\left(v_{\alpha \alpha}^{1} v_{n n}^{1}-\left(v_{\alpha n}^{1}\right)^{2}\right)=0 \text { in } U
$$

We have deduced that $v^{1}, v^{2}$ is a solution to (7.11), (7.13) subject to the boundary conditions

$$
v^{1}=0, \quad v^{2}=0, \quad \text { on } \Sigma .
$$

To linearize the equations at $y=0$ observe that

$$
\begin{aligned}
v^{j}(0)=v_{\alpha}^{j}(0)=v_{\alpha \beta}^{j}(0) & =v_{n \alpha}^{1}(0)=v_{n}^{2}(0)=0, \\
1 & \leqslant \alpha, \beta \leqslant n-1, j=1,2 .
\end{aligned}
$$

For variations $\bar{v}^{-1}, \bar{v}^{2}$ of $F_{1}$ and $F_{2}$ we obtain

$$
\begin{aligned}
& L_{11} \bar{v}^{1}+L_{12} \bar{v}^{2}=\frac{\bar{v}_{n n n}^{1}}{v_{n n}^{1}(0)^{3}}+\frac{2}{v_{n n}^{1}(0)} \sum_{\alpha<n} \bar{v}_{n n \alpha}^{1}+\sum_{\alpha<n} \bar{v}_{\alpha \alpha}^{2}, \\
& L_{21} \bar{v}^{1}+L_{22} \bar{v}^{2}=\bar{v}_{n}^{2}-v_{n n}^{1}(0) \sum_{\alpha<n} \bar{v}_{\alpha \alpha}^{1},
\end{aligned}
$$

corresponding to $F_{1}$ and $F_{2}$ respectively where, as usual, only the highest order terms of the linearization are considered. The Dirichlet boundary conditions are

$$
\bar{v}^{-1}=0, \quad \bar{v}^{2}=0 .
$$


A choice of the weights

$$
\begin{array}{lll}
s_{1}=0, & t_{1}=3, & r_{1}=-3, \\
s_{2}=-1, & t_{2}=2, & r_{2}=-2,
\end{array}
$$

where $s_{1}, s_{2}$ correspond to (7.15), (7.16), $t_{1}, t_{2}$ to $\bar{v}_{1}, \bar{v}_{2}$, and $r_{1}, r_{2}$ to the respective boundary conditions $\bar{v}^{1}=0$ and $\bar{v}^{2}=0$ reveals this boundary value problem to be coercive [ADN2]. Again invoking [Mo, p. 271], we see that the functions $v^{j}$ are analytic in $U \cup \Sigma$ near $y=0$. It follows, since $x_{n}=v_{n}^{1}$, that $\Gamma$ is analytic near $x=0$.

\section{BIBLIOGRAPHY}

[ADN1] S. Agmon, A. Douglis and L. Nirenberg, Estimates near the boundary for solutions of elliptic partial differential equations satisfying general boundary conditions. I, Comm. Pure Appl. Math. 12 (1959), 623-727.

[ADN2] __ Estimates near the boundary for solutions of elliptic partial differential equations satisfying general boundary conditions. II, Comm. Pure Appl. Math. 22 (1964), 35-92.

[Al] H. Alt, $A$ free boundary problem associated with the flow of groundwater, Arch. Rational Mech. Anal. 64 (1977), 111-126.

[An-F] R. F. Anderson and A. Friedman, A quality control problem and quasi-variational inequalities, Arch. Rational Mech. Anal. 63 (1976), 205-252.

[Ba1] C. Baiocchi, Su un problema a frontiera libera connesso a questioni di idraulica, Ann. Mat. Pura Appl. 92 (1972), 107-127.

[Ba2] __ Free boundary problems in the theory of fluid flow through porous media, Proc. Internat. Congr. Math. (Vancouver, 1974), Vol. II, Canad. Math. Congress, 237-343.

[Ba3] __ Problèmes à frontière libre et inéquations variationnelles, C. R. Acad. Sci. Paris 283 (1976), 29-32.

[Ben-F] A. Bensoussan and A. Friedman, Nonzero-sum stochastic differential games with stopping times and free boundary problems, Trans. Amer. Math. Soc. 231 (1977), 275-327.

[Ben-Li1] A. Bensoussan and J. L. Lions, Problèmes de temps d'arrêt optimal et inéquations variationelles paraboliques, J. Appl. Anal. 3 (1973), 267-294.

[Ben-Li2] __, Temps d'arrêt optimal et contrôle impulsionnel. Vol. I. (to appear).

[Be-Br] H. Berestycki and H. Brezis, Sur certains problèmes de frontière libre, C. R. Acad. Sci. Paris 283 (1976), 1091-1094.

[Br1] H. Brezis, Problèmes unilatéraux, J. Math. Pures Appl. 51 (1972), 1-168.

[Br2] _ Multiplicateur de Lagrange en torsion elastroplastique, Arch. Rational Mech. Anal. 41 (1971), 254-265.

[Br-D] H. Brezis and G. Duvaut, Ecoulment avec sillage autour d'un profile symètrique sans incidence, C. R. Acad. Sci. Paris 276 (1973), 875-878.

[Br-K] H. Brezis and D. Kinderlehrer, The smoothness of solutions to nonlinear variational inequalities, Indiana J. Math. 23 (1974), 831-844.

[BR-S1] H. Brezis and G. Stampacchia, Sur la régularité de la solution d'inéquations elliptiques, Bull. Soc. Math. France 96 (1968), 153-180.

[Br-S2] _ The hodograph method in fluid-dynamics in the light of variational inequalities, Arch. Rational Mech. Anal. 61 (1976), 1-18.

[C1] L. A. Caffarelli, The smoothness of a free boundary in a filtration problem, Arch. Rational Mech. Anal. 63 (1976), 77-86.

[C2] __ The regularity of elliptic and parabolic free boundaries, Acta Math.

[C3] _ The local regularity of a phreatic surface, Arch. Rational Mech. Anal. (to appear).

[C-R1] L. A. Caffarelli and N. M. Riviere, Smoothness and analyticity of free boundaries in variational inequalities, Ann. Scuola Norm. Sup. Pisa Sci. Fis. Mat. 3 (1976), 289-310.

[C-N2] __ Asymptotic behavior of free boundaries at their singular points, Ann. of Math. (to appear).

[C-N3] On the lipschitz character of the stress tensor when twisting an elastic plastic bar, Arch. Rational Mech. Anal. (to appear). 
[D] G. Duvaut, Resolution d'un probleme de Stefan (Fusion d'un bloc de glace a zero degré), C.R. Acad. Sci. Paris 276 (1973), 1461-1463.

[Fr-Ber] L. Fraenkel and M. Berger, A global theory of steady vortex rings in an ideal fluid, Acta Math. 132 (1974), 13-51.

[Fre1] J. Frehse, On the regularity of the solution of a second order variational inequality, Boll. Un. Mat. Ital. 6 (1972), 312-315. MR 47 \#197.

[Fre2] 279-288.

[Fre3] __ On Signorini's problem and variational problems with thin obstacles, Ann. Scuola Norm. Sup. Pisa Sci. Fis. Mat. 4 (1977), 343-362.

[F1] A. Friedman, On the regularity of the solutions of non-linear elliptic and parabolic systems of partial differential equations, J. Math. Mech. 7 (1958), 43-60.

[F2] __ The Stefan problem in several space variables, Trans. Amer. Math. Soc. 133 (1968), 51-87.

[F3] __, Analyticity of the free boundary for the Stefan problem, Arch Rational Mech. Anal. 61 (1976), 97-125.

[F4] , On the free boundary of a quasi variational inequality arising in a problem of quality control, Trans. Amer. Math. Soc. (to appear).

[F-J1] A. Friedman and R. Jensen, $A$ parabolic quasi-variational inequality arising in hydraulics, Ann. Scuola Norm. Sup. Pisa Cl. Sci. 2 (1975), 421-468.

[F-J2] _ Elliptic quasi-variational inequalities and application to a non-stationary problem in hydraulics, Ann. Scuola Norm. Sup. Pisa Cl. Sci. 3 (1976), 47-88.

[F-K] A. Friedman and D. Kinderlehrer, $A$ one phase Stefan problem, Indiana Univ. Math. J. 24 (1975), 1005-1035.

[G] P. Garabedian, Partial differential equations, Wiley, New York, 1964.

[Ge] C. Gerhardt, Regularity of solutions of nonlinear variational inequalities, Arch. Rational Mech. Anal. 52 (1973), 389-393.

[Gia-P] M. Giaquinta and L. Pepe, Esistenza e regolarità per il problema dell' area minima con ostacoli in n variabili, Ann. Scuola Norm. Sup. Pisa Sci. Fis. Mat. 25 (1971), 481-507. MR 46 \#4335.

[Giu] E. Giusti, Minimal surfaces with obstacles, CIME course on Geometric Measure Theory and Minimal Surfaces, Edizioni Cremonese, Rome, 1973, pp. 119-153.

[J.-Ser] H. Jenkins and J. Serrin, The Dirichlet problem for the minimal surface equation in higher dimensions, J. Reine Angew Math. 229 (1968), 170-187.

[Ka] C. Kahane, Analyticity of solutions of mildly singular integral equations, Comm. Pure Appl. Math. 18 (1965), 593-626.

[K1] D. Kinderlehrer, Variational inequalities with lower dimensional obstacles, Israel J. Math. 10 (1971), 339-348.

[K2] _ The coincidence set of solutions of certain variational inequalities, Arch. Rational Mech. Anal. 40 (1971), 231-250.

[K3] ___ How a minimal surface leaves an obstacle, Acta Math. 130 (1973), 221-242.

[K4] _ The free boundary determined by the solution as a differential equation, Indiana Univ. Math. J. 25 (1976), 195-208.

[K-N1] D. Kinderlehrer and L. Nirenberg, Regularity in free boundary problems, Ann. Scuola Norm. Sup. Pisa Cl. Sci. 4 (1977), 373-391.

[K-N2] _ The smoothness of the free boundary in the one phase Stefan problem, Comm. Pure Appl. Math. (to appear).

[K-N2] __ Analyticity at the boundary of solutions of nonlinear second order parabolic equations, Comm. Pure Appl. Math. (to appear).

[K-N-Sp] D. Kinderlehrer, L. Nirenberg and J. Spruck, (to appear).

[K-Sp] D. Kinderlehrer and J. Spruck, The shape and smoothness of stable plasma configurations, Ann. Scuola Norm. Sup. Pisa Sci. Fis. Mat. (to appear).

[K-S-1] D. Kinderlehrer and G. Stampacchia, A free boundary problem in potential theory, Ann. Inst. Fourier (Grenoble) 25 (1975), 323-344.

[K-S2] _ An introduction to variational inequalities and their applications,

[L1] H. Lewy, A note on harmonic functions and a hydrodynamical application, Proc. Amer. Math. Soc. 3 (1952), 11-113. 
[L2] , On the boundary behavior of minimal surfaces, Proc. Nat. Acad. Sci. U.S.A. 37

(1951), 103-110. MR 14, 168.

[L3] _ On minimal surfaces with partially free boundary, Comm. Pure Appl. Math. 4 (1952), 1-13. MR 14, 662.

[L4] - On the reflection laws of second order differential equations in two independent variables, Bull Amer. Math. Soc. 65 (1959), 37-58.

[L5] _ On a minimum problem for superharmonic functions, Proc. Internat. Conf. on Functional Analysis, Tokyo, 1969.

[L] _ On the nature of the boundary separating two domains with different regimes, Accad. Naz. Lincei, 1975.

[L-S1] H. Lewy and G. Stampacchia, On the regularity of the solution of a variational inequality, Comm. Pure Appl. Math. 22 (1969), 153-188. MR 40 \#816.

[L-S2] _ On existence and smoothness of solutions of some noncoercive variational inequalities, Arch. Rational Mech. Anal. 41 (1971), 241-253.

[Li-S] J.-L. Lions and G. Stampacchia, Variational inequalities, Comm. Pure Appl. Math. 20 (1967), 493-519. MR 35 \#7178.

[Mag] E. Magenes, Topics in parabolic equations: some typical free boundary problems, Proc. of NATO Advanced Study Institute, Liege, 1976.

[Ma1] S. Mazzone, Existence and regularity of the solution of certain nonlinear variational inequalities with an obstacle, Arch. Rational Mech. Anal. 57 (1974), 115-127.

[Ma2] __ Un problema di disequazioni variazionali per superficie di curvatura media assegnata, Boll. Un. Mat. Ital. 7 (1973), 318-329. MR 47 \#4109.

[Mi] M. Miranda, Frontieri minimali con ostacoli, Ann. Univ. Ferrara 21 (1971), 29-37.

[Mo] C. B. Morrey, Jr., Multiple integrals in the calculus of variations, Springer, New York and Berlin, 1966.

[Mu-S] M. K. V. Murthy and G. Stampacchia, $A$ variational inequality with mixed boundary conditions, Israel J. Math. 13 (1972), 188-224.

[P] J. Puel, Un probleme de valeurs propres non lineare et de frontiére libre, C. R. Acad. Sci. Paris 284 (1977), 861-864.

[Sc1] D. G. Schaeffer, Some examples of singularities in a free boundary, Ann. Scuola Norm. Sup. Pisa Cl. Sci. (4) 1 (1977), 133-144.

[Sc2] __, An example of the plasma problem with infinitely many solutions, Comm. in P. D. E. 2 (1977), 587-600.

[Sh] V. D. Shafranov, On magnetohydrodynamical equilibrium configurations, J. Exp. Theoret. Phys. (USSR) 33 (1957), 710-722.

[S-1] G. Stampacchia, Le problème de Dirichlet pur les équations elliptique du second order $\grave{a}$ coefficients discontinus, Ann. Inst. Fourier (Grenoble) 15 (1965), 189-258. MR 33 \#404.

[S-2] _ Variational inequalities, theory and applications of monotone operators, Proc. of

NATO Advanced Study Institute, Oderisi-Gubbio, 1969.

[S-3] __ Variational inequalities, Proc. Internat. Congr. of Math., Nice, 1970, 877-883.

[S-4], On the filtration of a liquid through a porous medium, Uspehi Mat. Nauk 29 (178) (1974), 89-101.

[S-5] _ Formes bilineaires coercitives sur les ensembles convexes, C. R. Acad. Sci. Paris 258 (1964), 4413-4416.

[Te1] R. Temam, Variational principles related to the equilibrium shape of a plasma in an axisymmetric torus, J. Plasma Physics (1976).

[Te2] __ A non-linear eigenvalue problem: the shape at equilibrium of a confined plasma, Arch. Rational Mech. Anal. 60 (1975), 51-73.

[Te3] _ Remarks on a free boundary problem arising in plasma physics, Comm. in P. D. E. 2 (1977), 563-585.

[Ti] T. W. Ting, Elastic-plastic torsion problem, Arch. Rational Mech. Anal. 24 (1969), 228-244.

School of Mathematics, University of Minnesota, Minneapolis, Minnesota 55455 